patients with obstructive jaundice.

Finally, the conference had a timely reminder from Dr Mary Dyson (London) of the possible risks of ultrasonic scanning. She described experiments in which chick embryos were irradiated at the head process stage. Certain dose parameters produced a significant increase in abnormalities, particularly of the central nervous system. At diagnostic levels no harmful results have been reported, but the field is not yet fully explored.

\section{CHEMISTRY}

\section{Asymmetry of C-F}

from a Correspondent

RECENT work on the fluorine n.m.r. spectra of silver trifluoracetate, $\mathrm{Ag}^{+}$ $\mathrm{CF}_{3} \mathrm{CO}_{2}^{-}$, by Waugh and his colleagues at MIT (J.Chem. Phys., 57, $2147 ; 1972)$ has revealed a remarkable lack of axial symmetry in the chemical shift tensor for the C-F bonds.

A typical C-F bond has avial symmetry, that is all directions perpendicular to the bond are equivalent. More specifically, vector properties such as a bond dipole moment contribution will lie parallel to the bond: likewise a second rank tensor associated with a property such as the optical polarizability is expected to have one of its principal directions parallel to the bond and the other two to be associated with equal principal values and consequently referring to all directions perpendicular to the bond. Such axial symmetry is a necessary property of a model of a bond in isolation, and would be expected to be realistic for real bonds in molecules, at least in the absence of $\pi$ electron or aromatic effects.

To understand the findings it must be remembered that an n.m.r. chemical shift, the quantity $\sigma$ in the energy expression, $-\hbar \gamma \mathbf{I} \cdot(1-\sigma) \cdot \mathbf{B}$, relates to the departure of the n.m.r. frequency for the substance being studied from that of the bare nucleus or other reference state. For fluorine, $\sigma$ may reach several hundred p.p.m. and is well known for many liquids. But for liquids the quantity observed is the mean value of $\sigma$, that is one-third of the trace (Spur or diagonal sum) of the tensor quantity. With very few exceptions these mean values are the only quantities that have been available. The fuller information could not be obtained from solid state spectra because it is normally obscured by the dipole-dipole coupling between nuclei; this is a much bigger effect than the chemical shift and leads to line widths of the order of $10 \mathrm{kHz}$.

Waugh and his colleagues used a special sequence of radiofrequency pulses to excite the resonance and to remove the effect of the dipole-dipole

coupling. The line widths were 0.5 $\mathrm{kHz}$ or less, thus enabling the details of the chemical shift to be made plain. In a single crystal of $\mathrm{Ag}^{+} \mathrm{CF}_{3} \mathrm{CO}_{2}{ }^{-}$, at least at $40 \mathrm{~K}$ where the $-\mathrm{CF}_{3}$ rotational motion has been frozen, each distinct $C-F$ bond in the unit cell may make a different angle with the magnetic flux density and consequently have a different effective chemical shift for the fluorine resonance. Six lines were resolved and measurements at several crystal orientations with respect to the magnetic field allowed the complete chemical shift tensor to be derived for each of the C-F bonds of the $-\mathrm{CF}_{3}$ group. There are small, yet real, differences between the bonds, but essentially the results indicate, for each C-F bond, that the principal values and principal axis directions of the $\sigma$ tensor are +73 p.p.m. along the bond, -75 p.p.m. in the CCF plane and +2 p.p.m. in the circumferential direction around the $-\mathrm{CF}_{3}$ group.

Such a tensor is clearly not of the expected axial type, which would require principal values in the ratios $+74:-37:-37$. This is surprising, for the electrons in the immediate vicinity of the nucleus normally dominate its chemical shift. Theoretical chemists will have to get to work to explain the lack of axial symmetry and the differences between the three bonds, and to find the reason why the principal direction is actually at an angle of $10^{\circ}$ to the C-F bond direction rather than strictly parallel to it.

\title{
Oncornaviruses and Breast Cancer
}

Two of the chief obstacles in the way of further investigations of the viruslike particles which have been detected in samples of human milks and may be human counterparts of mouse mammary tumour viruses are the lack of cultivated cells which can be productively infected or transformed by these milk particles, and the lack of cultures of human breast cells that produce the particles. At present these cells can be obtained only from human milk and there is no assay for their biological activity. The experiments reported in next Wednesday's Nature New Biology (January 10) by Keydar et al., however, may show the way to a method of obtaining reproducible cultures of human cells that support the replication of the human "milk virus".

Keydar and his colleagues co-cultivated primary human embryo cells and human breast cancer cells, and exposed other cultures of human embryonic cells to milk, from breast cancer patients, which had reverse transcriptase activity and presumably contained the milk particles. In nine out of a

\section{NUCLEAR PHYSICS}

\section{Exchange Effects}

EVIDENCE for the occurrence of substantial exchange effects in the elastic scattering of oxygen-16 nuclei from oxygen-18 nuclei is given in a recent issue of Physical Review Letters (29, 1683 ; 1972) by Gelbke and his colleagues of the Max-Planck-Institut für Kernphysik at Heidelberg.

It has long been known that, when a light nucleus (typically of mass less than 40 a.m.u.) is scattered from a target composed of similar light nuclei, the observed distribution in angle of the bombarding particles cannot be explained solely by an interaction in which the incident particle is affected by a combination of the Coulomb repulsion between the particles and a nuclear interaction simulated by a potential well with a real and imaginary part (an optical model potential). What the extra mechanism is or what quirk of the mechanism makes the observed angular distribution of elastically scattered heavy particle deviate from that predicted by the optical model has been the subject of considerable speculation. The work of Gelbke et al., however, eliminates some of the suggested possibilities and comes out in favour of an exchange contribution.

The telltale feature of anomalies of this kind in elastic scattering is their variation with the energy of the incident particle. The Heidelberg team studied the reaction of ${ }^{16} \mathrm{O}$ on ${ }^{18} \mathrm{O}$ at $24 \mathrm{MeV}$,

total of fifteen such cultures Keydar et al. detected the production of particles which contain 60-70S RNA and reverse transcriptase activity and have a buoyant density of $1.18 \mathrm{~g} \mathrm{ml}$. These are, of course, diagnostic properties of RNA tumour viruses. Furthermore, some of these cultures have been producing these particles for nine months whereas control cultures of human embryonic cells not exposed to milk or co-cultivated with cancerous breast tissue have not produced any detectable particles with the characteristics of RNA tumour viruses.

As Keydar et al. point out, the particles which are being produced in their cultures may have arisen from the cancer cells and may be involved in the aetiology of breast cancer. On the other hand, they may be contaminants or they may have arisen in the embryonic cells. These second alternatives, however, are less likely and attempts are under way to characterize the particles produced in the cultures and establish their relationship, if any, with virus-like particles in human milks. 
$28 \mathrm{MeV}$ and $32 \mathrm{MeV}$-energies close to and slightly above the Coulomb barrier for the interaction of these oxygen nuclei. Gelbke and his colleagues found that at these energies the angular distribution of the scattered particles has two distinct minima in intensity between $90^{\circ}$ and $150^{\circ}$; these occurred at approximately the same angle regardless of the energy of the incident particle. This observation immediately rules out explanations of the anomalous behaviour based on the absorption of the incident particle by the nucleus to different extents depending on its angular momentum relative to the scattering nucleus. Thus the anomalous behaviour cannot be explained by an optical model that contains a term which explicitly depends on the angular momentum of the particles, although observations at one energy can be adequately described by such a model.

Gelbke et al. think that the incident ${ }^{16} \mathrm{O}$ nucleus picks up the two least bound neutrons in an ${ }^{18} \mathrm{O}$ nucleus and imparts sufficient momentum to the ${ }^{16} \mathrm{O}$ nucleus thus formed for it to emerge from the target. This ${ }^{16} \mathrm{O}$ is, of course, indistinguishable from a normally scattered ${ }^{16} \mathrm{O}$ nucleus.

The experimental angular distributions are fitted by a model which includes contributions from such an exchange mechanism and, as well as reproducing each individual measured distribution at the three energies, the variation with energy is also satisfactorily reproduced. Minor differences in the theoretical fits to the experimental points at the three energies are attributed to the fact that the probability of the incident ${ }^{16} \mathrm{O}$ nucleus picking up the two neutrons from ${ }^{18} \mathrm{O}$ varies with energy.

\section{RADIATION DAMAGE}

\section{Precipitate Instability}

from our Materials Science Correspondent IT is not at all surprising that an unprecedented research effort is being devoted to a form of radiation damage that is specific to fast reactors for, even though nuclear power, under pressure from environmentalists, is unfashionable, those persons responsible for national energy policy know perfectly well that in the long run most power must come from nuclear reactors, and the fast reactor in particular.

Radiation damage in fast reactors is a consequence of the fast neutrons and the high fuel temperatures: fuel swelling is caused by the formation of voids, which are themselves formed by the selective agglomeration of excess lattice vacancies resulting from neutron damage. When a neutron starts a collision sequence which causes a number of atoms to be knocked out of the crystal lattice, equal numbers of vacancies and interstitials result. If, as is all too apt to happen, the interstitial atoms migrate faster than the vacancies, then the former quickly disappear at "sinks" and the vacancies are left high and dry; they are then likely to combine to form microscopic cavities or voids, and the fuel or the material of the fuel envelope swells dangerously. This has been a serious problem, but because of extensive research since 1967 it is now well on the way to solution.

One of the principal remedies is to use alloys containing a fine dispersion of precipitates; migrating interstitials are in some way arrested when they impinge on a precipitate particle, and are held captive there until they are neutralized by a wandering vacancy. So, in the end, almost all the interstitials recombine with vacancies and few vacancies are left to form voids. Thus, some nickel-base alloys of the Nimonic type have been found particularly suitable for the manufacture of fuel cans, for the fine precipitates of $\gamma^{\prime}$ (ordered $\mathrm{Ni}_{3} \mathrm{Al}$ ) impede swelling in the manner described. For this purpose, it is necessary for the precipitates to be particularly finely distributed (to increase the probability of interstitial capture), and to stay that way in service. It is this last requirement which is difficult, because all precipitate populations are subject to the law of Ostwald ripening -that is, operation of the Matthew Principle, or the growth of the large at the expense of the small.

A group of investigators at Harwell have now systematically examined, both theoretically and experimentally, the behaviour of precipitate populations in a radiation field. The Matthew Principle is counteracted by the steady destruction of precipitates by neutron damage, and the diffusion of solute atoms into the matrix at a rate which is itself accelerated by the presence of excess vacancies. From their theoretical calculations, Nelson, Hudson and Mazey (J. Nucl. Mater., 44, 318 ; 1972) find that bigger particles redissolve faster than small ones, and that this is true whichever of two alternative mechanisms of resolution operates.

The final conclusion is that in the presence of steady neutron irradiation, large particles dissolve preferentially but small particles on balance still grow, so that a dynamic equilibrium is established and most particles finish up close to a preferred size, which is quite small. Irradiation thus effectively neutralizes Ostwald ripening. This conclusion is confirmed by experimental observations on a Nimonic-type alloy containing coherent $\gamma^{\prime}$ particles, but not by observations on another kind of nickel alloy containing incoherent precipitates. The last of these are subject to normal coarsening, because (it seems) one of the mechanisms for irradiation induced resolution of precipitates, which requires mass transport across the particle/matrix interface, does not work satisfactorily across incoherent (high energy) interfaces.

\section{BL LAC OBJECTS}

\section{Link with Galaxies?}

by our Cosmology Correspondent

BL LACERTAE is the archetype of a class of interesting extragalactic objects. These BL Lac objects seem to be exceedingly compact, and are in many ways reminiscent of QSOs, with the important difference that they have no spectral lines and thus no measured redshifts. Although only half a dozen of the objects are known, and perhaps no more than three can be confidently said to be of the same family as BL Lac itself, they have recently been the subject of intensive study because they may be related to QSOs.

One interesting speculation-it is no more at present-is that BL Lac objects may be blueshifted QSOs; their flat,

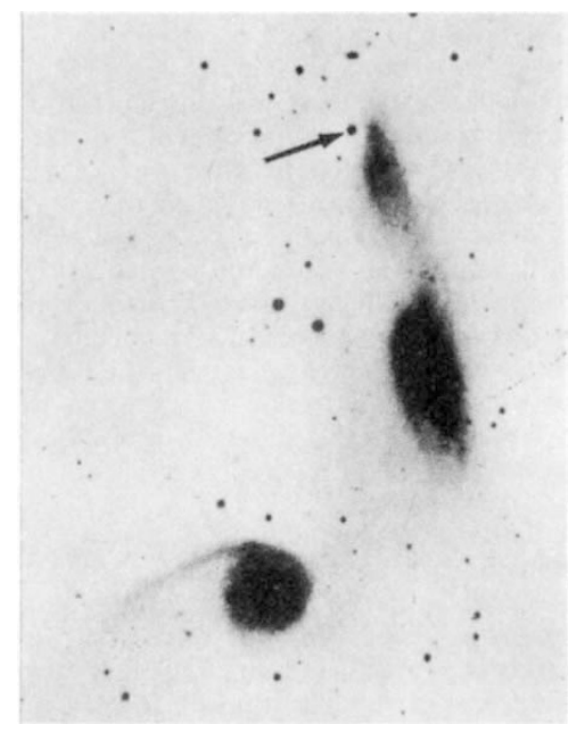

NGC 2993 (bottom), NGC 2992, and Weedman 2 (arrow). (Plate obtained by H. C. Arp.)

line-free spectra could well represent a region "normally" seen in the infrared. This idea is important, even though the sample of BL Lac objects is so small, because the definite detection of even one blueshifted QSO would have a significant bearing on the "redshift/distance relation" of QSOs. There is mounting evidence that the redshift of QSOs cannot be explained simply as a Doppler effect of motion in an expanding universe, and that part of the redshift, in some QSOs at least, must arise in some other way.

Paramount among the reasons underlying this view is the discovery of some 